\title{
Optimized Algorithm for Target Tracking using Classification Information based Association
} Filter

\author{
Anita Thite, Arun Mishra
}

\begin{abstract}
In last few decades, multiple target tracking fetches quite attention to the researchers for object localization and monitoring target trajectories which has become one of the most used technique in the area of visual tracking, traffic monitoring, air surveillance system, robotics and vision. On the basis of $S-D$ assignment algorithm, a new algorithm for tracking multiple targets in presence of clutter is designed. By considering target classification information received as special feature from target scan report, cost coefficients of dynamic assignment matrix are modified accordingly using joint probabilistic data association filter. The tracking results get improved with the use of target class and kinematic features information where the association costs are similar for different targets. With the help of the information collected in current scan the classifier output is dynamically updated to incorporate new target classes to be used future scans. Simulation results show that new algorithm can attain competitive tracking performance with distributed computational load by utilizing target classification information into dynamic multidimensional assignment algorithm. The main contribution of this paper is the development of new target tracking method based on IMM filter which generate dynamic classifier to incorporate target features information. This additional information about targets present in current scan helps to take future scans data association decisions.
\end{abstract}

Keywords: Multiple Target Tracking (MTT), Air Surveillance Systems, Data Association (DA), Multiple Hypotheses Tracking (MHT), Interactive Multiple Models (IMM)

\section{INTRODUCTION}

With the development of modern network centric warfare, the emergence of multiple sensors and track while scan infrared has significant impact on the design of target tracking systems that traditionally dependent on radars. Targets in real time tracking scenarios may be detected and continuously monitored by their reflected signals emitted from radar, sonar or use of various types of sensor networks. Multiple target tracking and its relevant research aspects are discussed in this section. Target tracking involves monitoring the position and movements of multiple targets at every time step.

Revised Manuscript Received on October 30, 2019.

* Correspondence Author

Anita Thite, Computer Science and Engineering Department, Defence Institute of Advanced Technology, Pune, India

Arun Mishra, Computer Science and Engineering Department, Defence Institute of Advanced Technology, Pune, India

(C) The Authors. Published by Blue Eyes Intelligence Engineering and Sciences Publication (BEIESP). This is an open access article under the CC-BY-NC-ND license http://creativecommons.org/licenses/by-nc-nd/4.0/
The varying numbers of indistinguishable targets are moving randomly in a given region and multiple sensors collects data reports from Field of View (FOV) containing positions of moving target at random intervals [1]. This information is in the form of measurements from an unknown number of targets and received at each radar scan. Target tracking requires each measurement received to be associated with an existing or new target track. These measurement-to-track associations are used to estimate the state trajectories of a moving target. In addition to target-originated measurement there exist false alarms which are the measurements due to noise and clutter. In addition to tracking inaccuracy due to false alarms, there is also uncertainty related to the origin of measurement, it is not always possible that tracking algorithm use true measurement that is originated from target of interest. Thus one of the most important stages in multimarket tracking problems is measurement-to-track data association due to presence of more than one target or extraneous object in same locality. The problem becomes more challenging when targets are closely spaced and conflicting situation occur where several unknown number of targets crossed and coalescences. A well-known tracking method to handle such conflicting tracking scenario in presence of clutter is Joint Probabilistic data Association (JPDA) filter. It handles measurement-to-track uncertainty by calculating association probabilities for each validated measurement [1]. In JPDA filter, each target's state, conditioned on the past scan, are assumed to be distributed independently and probability of each assignment is incorporated into state estimates. In this paper an equivalent filter to the JPDA with target classification information is used in proposed algorithm design.

In a general sense, a solution for measurement-to-track data association performs with k-best solutions in standard assignment problem called Murty's algorithm. The proposed algorithm design is formulated as a constrained optimization problem, where the cost function is a formulated based on PDA state estimator. But through simulations, we find that traditional 2-D assignment method which performs dynamic association for target tracking is not performing satisfactorily and at the same time its computational cost is too large, because of , the projective transforms approaches do not deem that the target tracking as a stochastic estimation problem.

In fact besides the target location information, sensors can send more information of targets e.g target classification, target features. 
A target tracking algorithm, which incorporated such information in tracking process, was presented in Wang [1] it works for targeting tracking based on address of image, on given longitude as well as latitude values. This approach mainly works based on event localization approach.

This paper, we describe a new tracking algorithm which utilizes the target feature information to deal with data association with false alarm. The algorithm is based on prioritized S-D assignment formulation. By considering the target classification information as special feature of IR sensors, the cost coefficients for association process are modified using a joint probabilistic model. In last step we update dynamic classifier to include new classes and corresponding features based on multiple motion models considered. The simulation results show that the proposed algorithm has better performance than traditional assignment with less computational cost.

\section{Methodology}

The proposed target tracking algorithm gives the solution to the inseparable problem in tracking system that is data association. The intelligent target tracking and monitoring involve combination of data acquired by multiple sensors, which makes observation of environment under its field of view (FOV) in order to detect actual targets and other objects. For designing the proposed tracking system, Probabilistic Data Association Filter (JPDAF) and error model Kalman filer are used for state estimation, target classifier is designed to take advantages of extra features of target that help in formation of tracks. The target classifier considered in this scheme is updated dynamically as tracking process evolves with next scans. Figure 1 show the proposed tracking methodology based on target classification information. The algorithm considers IMM models which include both maneuvering and non-maneuvering motion of targets. Initially pure IMM methods are applied along with tracking algorithm with prior assumptions. Proposed tracking algorithm shows improvements in assignment process from third and above scans. In last phase, the algorithm create new tentative classes according to target behavior (motion) and update dynamic classifier to include these new classes and corresponding features in future scans.

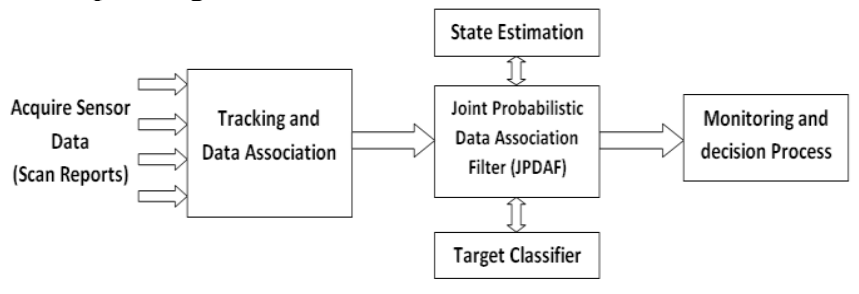

Fig. 1. Proposed Tracking Methodology

\section{A. Proposed Mathematical Model}

Let assume there are $\mathrm{T}$ targets in dense clutter environment with clutter density, at time $\mathrm{t}(\mathrm{k})$, tracker obtains total $\mathrm{M}$ measurements from each $\mathrm{s}=1,2, \ldots ., \mathrm{S}$ passive sensors, which include position, velocity and other features of potential targets. In particular, the state of nth target is given by

$$
x_{n+1}^{T} y=(x \& y)_{n+1}
$$

Target dynamics and measurement are given by [1],

$$
\begin{aligned}
& x(k)= \phi(k, k-1) x(k-1)+G(k, k-1) \omega(k-1) \\
& \text { where } x(k) \rightarrow \text { System State, } \\
& \phi(k, k-1) \rightarrow \text { State Transition Matrix, } \\
& \omega(k-1) \rightarrow \text { Process Noise } \\
& G(k, k-1) \rightarrow \text { Excitation Matrix } \\
& z(k)=H(k) x(k)+v(k)
\end{aligned}
$$$$
\text { where } z(k) \rightarrow \text { Measurement Vector }
$$$$
H(k) \rightarrow \text { Observation Vector }
$$$$
v(k) \rightarrow \text { Measurement Noise }
$$

The target tracking system recursively estimates the target state $\mathrm{x}(\mathrm{k})$ given in equation (2). The tracking process is stochastic in nature and therefore it finds probability density function (pdf) of the target states as time evolves. The target state usually represented by positions and velocities of objects as given in equation (1) and in order to estimate the states measurements vector are available which is received in the form of data scan reports from sensors as given in equation (3) The tracking process always based on two models: the target motion model and measurement model. Motion model describes the target motion dynamics in terms of velocity, acceleration and turn rate whereas measurement model captures the target's state and covariance in terms of measurement matrix with 2-D or 3-D coordinates [3].

\section{B. Target tracking and data association}

Sensors send the data scan reports consists of multiple measurements based on target dynamics. It receives measurements both due to actual targets and measurement noise. Data association process deals with problem of selecting the measurements that most probably initiated from the actual target and eliminate the false measurements and clutter due to noise.

If the correct measurement is not associated with actual target then tracking results incurs error which results in miscorrelation.

A new target track get initiated based on initial state estimates of all possible tracks along with associated state covariance matrix. There is always possibility of false track being generated due to presence of noisy measurements and multiple targets exist in a scenario. The track initiation technique is based on the logic that initiate new track only if $\mathrm{M}$ detection out of $\mathrm{N}$ scan in a gate can be useful. After the formation of track, the measurement selection is carries out by monitoring and decision process based on mathematical model described in section 2.1 to determine potential candidate measurements for corresponding track update.

\section{Multi-Dimensional (Multiframe) Assignment}

We consider a formal definition of the classical assignment problem in target tracking as follows: 
Minimize

$$
\mathbf{Z}=\sum_{i=1}^{n} \sum_{j=1}^{n} c_{i, j} x_{i, j}
$$

Subject to $\sum_{i=1}^{\mathrm{n}} x_{i, j} \quad{ }_{i=1 \ldots n} \sum_{j=1}^{\mathrm{n}} x_{i, j} \quad{ }_{j=1 \ldots n} \mathbf{x}_{\mathrm{i}, \mathrm{j}} \in\{\mathbf{0}, \mathbf{1}\}$

where $\mathrm{X}=\left[\mathbf{x}_{\mathbf{i}, \mathbf{j}}\right]$ denotes a feasible assignment and $\mathrm{C}=\left[\boldsymbol{c}_{\boldsymbol{i}, \boldsymbol{j}}\right]$ represents the cost matrix,

Assume, $\boldsymbol{c}_{\boldsymbol{i}, \boldsymbol{j}}$ is the log-likelihood score of associating the $\mathrm{i}^{\text {th }}$ measurement to the $\mathrm{j}^{\text {th }}$ track and $\mathrm{Z}$ is the cost of a specific data association $\mathrm{X}$ given cost matrix $\mathrm{C}$.

This problem of association is called as data assignment problem in optimization literature [4].

The decision making process in case of conflicting scenarios in tracking algorithm is formulated as Assignment problem and solution to this assignment problem will help us to resolve conflicts and get output estimated tracks precisely. We formulated this process as S-D assignment problem where one track list is considered and (S-1) measurement lists are available for data association.

\section{Murty's Method}

S-D assignment problem can be solved by determining a ranked set of solutions based on assigned probabilistic weights. The ranked assignments consistent with value within the classical linear assignment drawback will be resolved by Murty's methodology [12]. AN assignment algorithmic rule partitions the set of doable assignments supported value adore every assignment combine, where, the best assignment is calculated employing a new reweighted improvement technique for every partition. As for any improvement issues, the assignment drawback will be generalized to rank the ' $\mathrm{K}$ ' best assignments in non-decreasing order of value. Similarly, during this methodology a reweighting technique wherever the set of doable assignments is partitioned off into at the most (n-1) disjoint subsets [12]. The auction algorithmic rule is employed to search out the most effective pairs for every set leading to $\mathrm{AN} \mathrm{O}(\mathrm{Kn} 4)$ complexness for locating the $\mathrm{K}$ best solutions. Basic steps that describe the Murty's methodology area unit given as:

1. Given the assignment matrix Ai, which include cost for measurement-to-track association for each individual feasible measurement.

$C^{*}=\left(\begin{array}{cccccccccc} & T_{1}^{c} & T_{2}^{c} & T_{3}^{\prime} & T_{4}^{\prime} & T^{\text {new }} & T^{\text {new }} & T^{\text {new }} & T^{\text {new }} & T^{\text {new }} \\ M_{1} & 0.30 & 4.70 & 4.92 & 4.91 & 5 & \infty & \infty & \infty & \infty \\ M_{2} & 4.90 & 0.20 & 4.91 & 4.99 & \infty & 5 & \infty & \infty & \infty \\ M_{3} & 4.10 & 4.90 & 4.89 & 4.93 & \infty & \infty & 5 & \infty & \infty \\ M_{4} & 4.01 & 4.89 & 4.89 & 4.96 & \infty & \infty & \infty & 5 & \infty \\ M_{5} & 4.20 & 4.79 & 4.87 & 4.97 & \infty & \infty & \infty & \infty & 5 \\ M_{6}^{d} & 10 & \infty & \infty & \infty & 0 & 0 & 0 & 0 & 0 \\ M_{7}^{d} & \infty & 10 & \infty & \infty & 0 & 0 & 0 & 0 & 0 \\ M_{8}^{d} & \infty & \infty & 10 & \infty & 0 & 0 & 0 & 0 & 0 \\ M_{9}^{d} & \infty & \infty & \infty & 10 & 0 & 0 & 0 & 0 & 0\end{array}\right)$

Fig. 2. Top five assignment by Murty's Method

\section{Find the most effective resolution mistreatment Auction} (Hungarian) algorithmic rule.

3. Find the challenger resolution by:

a. Specific to the $2 \mathrm{D}$ best resolution because the resolution of variety of best resolution assignment sub issues. b. Note the answer to every of those sub issues mistreatment Hungarian algorithmic rule.

c. The resolutions which supplies most reward (minimum cost) area unit future best solution. Minimum cost will be the negative log-likelihood score of associating the $i^{\text {th }}$ measurement to the $\mathrm{j}^{\text {th }}$ track

4. Repeat the procedure for further more solutions

Table I: Top five Assignment using S-D Assignment

\begin{tabular}{|c|c|c|c|c|c|}
\hline $\begin{array}{l}\text { Assignments } \\
1 \quad \text { Tr Tracks } \\
\end{array}$ & $\operatorname{Tr}_{1}{ }^{\mathrm{C}}$ & $\operatorname{Tr}_{2}{ }^{\mathrm{C}}$ & $\operatorname{Tr}_{3}{ }^{I}$ & $\operatorname{Tr}_{4}{ }^{I}$ & $\operatorname{Tr}^{\mathrm{nev}}$ \\
\hline a1 & $\mathrm{M}_{1}$ & $\mathrm{M}_{2}$ & M5 & M3 & M4 \\
\hline a2 & $\mathrm{M}_{1}$ & $\mathrm{M}_{2}$ & M5 & M4 & M3 \\
\hline a3 & $\mathrm{M}_{1}$ & $\mathrm{M}_{2}$ & M3 & M4 & M5 \\
\hline a4 & $\mathrm{M}_{1}$ & $\mathrm{M}_{2}$ & M3 & M5 & M4 \\
\hline a5 & $\mathrm{M}_{1}$ & $\mathrm{M}_{2}$ & M4 & M5 & M3 \\
\hline
\end{tabular}

Assume that there are two confirmed tracks $\mathrm{T}_{\mathrm{r} 1}{ }^{\mathrm{c}}$ and $\mathrm{T}_{\mathrm{r} 2}{ }^{\mathrm{c}}$, two tentative tracks $\mathrm{T}_{\mathrm{r} 3}{ }^{\mathrm{I}}$ and $\mathrm{T}_{\mathrm{r} 4}{ }^{\mathrm{I}}$, one newly initialized but yet to be confirmed track and five measurements $\mathrm{M}_{1}, \mathrm{M}_{2}, \mathrm{M}_{3}, \mathrm{M}_{4}$ and $\mathrm{M}_{5}$, the corresponding cost matrix is given as $\mathrm{C}^{*}$.

The top five assignment generated based on the cost matrix $\mathrm{C}^{*}$ is given in figure 3 . It denotes the pair of indices corresponding to particular measurement and its associated track respectively.

The top 5 assignments generated based on the above cost matrix are:

$$
\begin{aligned}
& a_{1}^{+}=\{(1,1),(2,2),(3,4),(4,8),(5,3),(6,5),(7,6),(8,7),(9,9)\} \\
& a_{2}^{+}=\{(1,1),(2,2),(3,7),(4,4),(5,3),(6,5),(7,6),(8,9),(9,8)\} \\
& a_{3}^{+}=\{(1,1),(2,2),(3,3),(4,4),(5,9),(6,5),(7,6),(8,7),(9,8)\} \\
& a_{4}^{+}=\{(1,1),(2,2),(3,3),(4,8),(5,4),(6,5),(7,6),(8,7),(9,9)\} \\
& a_{5}^{+}=\{(1,1),(2,2),(3,7),(4,3),(5,4),(6,5),(7,6),(8,8),(9,9)\}
\end{aligned}
$$

Fig. 3. Data Association results (top five) by using the Standard Murty's Algorithm

Tracking targets always involves association of set of mk approved estimations gotten by gating tests as portrayed in segment 2 with realized targets set T. The occasion $\phi \mathrm{ij}$ is characterized as estimation $\mathrm{j}$ began from objective I. Relating estimation to target affiliation probabilities to these occasions are determined together crosswise over focuses with assistance of Probabilistic information affiliation channel [2][3]. A mistake model Kalman channel (EKF) calculation is connected to every one of the approved estimations. Kalman channel ascertains the covariance network and a Kalman gain for each approved estimations which relies upon The entryway likelihood PG, size of the approval area, number of approved estimations mk, A zero-mean ordinary circulation with contention__i(k) and covariance, the likelihood I of occasion $\phi i j$. Track gets refreshed with the related estimation which is picked to be a weighted normal of every approved estimation where the probabilities are the loads.

Only the last estimation is utilized no history will be considered and amassed for next sweep and each objective state is demonstrated by its state directions and covariance by straight unique estimation model. 
Further, the quantity of targets is thought to be known at each output and the outcome is a lot of weighted midpoints of identification likelihood. Generally it considered just direct models but In order to deal with nonlinear systems all modern tracing systems use Interactive Multiple Models (IMM) to incorporate all motion models and recover erroneous matches [10].

\section{IMM ESTIMATOR FILTER}

Targets can move with maneuvering and non-maneuvering motion. Uniform motion with constant velocity which is always straight is known as non-maneuvering motion whereas accelerated and turning movements are maneuvering. Usually tracking with accurate target's state estimation requires suitable target motion model [11]. A single motion model is no longer useful to track maneuvering targets because targets acceleration and turn cannot track accurately with only single motion model, instead multiple models are used to describe the motion of the target.

In IMM estimator, multiple motion model filters are used for effective tracking of maneuvering and non-maneuvering targets [12]. In IMM, various possible target motion models, the trajectory movements, maneuver patterns and turn conditions are handled by a series of multiple kalman filters. IMM calculates the state estimates and covariance from different motion model separately by applied Kalman filters and its weighted sum is taken as the final state estimation. Transition probability decides whether to switch the mode from one target motion model to another suitable model. Thus the IMM algorithm is used to estimate target motion state accurately and solve problem of the manoeuvring target tracking [13]. Various dynamic motion models, constant velocity model and variable velocity, constant turn and acceleration models coupled with JPDA filer are considered to capture dynamic motion of target.

\section{Formulation OF TARgET ClASSIFIER}

The target tracking algorithms always use the target motion model and target dynamics parameters. It cannot easily incorporate with other feature information about target that is received with data scan reports. The extraneous information about target such as target length and radar cross section, target type can be obtained along with the enhancement of sensor resolution. This information can utilize to assist tracking. The proposed algorithm incorporates this initial feature information about targets in the target classier matrix. This classifier matrix is used to update the association probabilities of measurement- to track in order to make best use of domain knowledge. The class feature measurement likelihoods are calculated for each track and threshold value $>=80 \%$ is used to decide whether target belongs to same class or not. This feature measurement likelihood is used into the construction of the distribution function in IMM filters. As more and more class feature information get incorporated in tracking algorithm and target tracks match the particular class with higher probability, it apparently enhance the classification ability. These reasons motivate our choice of the feature measurement likelihood for developing the tracking algorithm.

In order to classify the targets, we modeled the class feature measurement likelihood calculated by IMM and it gets updated at the end of each scan, called classification estimates. These dynamic classification estimates are used to in future scans. Our proposed method uses the multiple models with IMM techniques and combines tracking and classification results to improve final tracking estimates.

\section{A. Algorithm for Target Tracking using Proposed IMM with Class information (PIMMwithCI) formulation the target cluster}

Let the nExtistingTargets are detected and present in current scenario

For $n=1:$ nExtistingTargets

Step1: Formulate tracks for all nExtistingTargets and perform Gating Test

Step2: At time t for all nExtistingTargets

Run Proposed IMM with Class information PIMMwithCI

If Target $\neq$ Associated (Target not associated with current measurement due to conflict scenario or temporarily missed detection)

If T_LifeTime $>0$

Run PIMMwithCI algorithm for state prediction and track update

$$
\text { T_LifeTime }=\text { T_LifeTime-1 }
$$

\section{END}

Else If Target $=$ Associated

Implement IMM Track Update

T_LifeTime $=$ T_LifeTime-1

Goto step 3

END

Else (LifeTime $=0$ )

Continue; (Target track is deleted or target get disappeared)

END

Step3: Ouput target's state estimate and merge covariances. For $n=1$ : nNewlyCreatedTargets (new target is detected and corresponding measurement get received)

Use current measurement as initial position

Initialise its T_LifeTime $=0$

Repear step 1-3

END

\section{B. Algorithm for Proposed IMM - PIMMwithCI and Formulation the target cluster}

Step 1: Let Mi denote the set of all target motion modes connected to class from individual starting points

$\mathrm{M} 1=\{\mathrm{m} 1\}$,

$\mathrm{M} 2=\{\mathrm{m} 1, \mathrm{~m} 2, \mathrm{~m} 3\}$ and

$\mathrm{M} 3=\{\mathrm{m} 1, \mathrm{~m} 2, \mathrm{~m} 3, \mathrm{~m} 4, \mathrm{~m} 5\}$.

Step 2: Calculation of the mixing probability: The mixing probability is given by

$$
\mu_{k-1 \mid k-1}^{i \mid j}=\frac{1}{\bar{c}_{j}} p_{i j} \mu_{k-1 \mid k-1}^{i} \quad i, j=1, \ldots, r
$$
where $\mathrm{Pij}=\mathrm{p}\left(m_{k}^{j} \mid m_{k-1}^{i}\right)$ is the mode transition probability and 
$\bar{c}_{j}=\sum_{i=1}^{r} p_{i j} \mu_{k-1 \mid k-1}^{i} \quad j=1, \ldots, r$

Stage 3: Mixing: The mean objective state and the covariance network for the jth mode-coordinated channel are given by given examples

Stage 4: Mode-coordinated sifting: The objective states and the covariance in stage 2 and stage 3 are utilized as contribution to the progress mode-coordinated channel mj $\mathrm{k}$ with estimation zk. The mode probability worth chooses to change to another movement model and define new model set or bunch.

Stage 5: Transition Mode likelihood update: The mode likelihood update is given by record set for forward group just as in reverse bunch.

Stage 6: Estimate: Finally the gauge and comparing covariance grid are discovered utilizing restricted coordinating of model likelihood. It is given by

$$
\begin{aligned}
\hat{\mathbf{x}}_{k \mid k} & =\sum_{i=1}^{T} \mu_{k \mid k}^{j} \hat{\mathbf{x}}_{k \mid k}^{j} \\
P_{k \mid k} & =\sum_{i=1}^{r} \mu_{k \mid k}^{j}\left\{P_{k \mid k}^{j}+\left[\hat{\mathbf{x}}_{k \mid k}^{j}-\hat{\mathbf{x}}_{k \mid k}\right]\left[\hat{\mathbf{x}}_{k \mid k}^{j}-\hat{\mathbf{x}}_{k \mid k}\right]^{T}\right\}^{\text {Kall }}
\end{aligned}
$$

Step 7: return estimated target state as $\mathrm{Pk} \mid \mathrm{k}$

C. Data Association using Target classifier information

The flowchart of data association process with Joint Probabilistic Data Association Filter (JPDAF) and proposed IMM based algorithm is given in figure 4. Measurement model and motion model are used to find estimated target states and covariance at each data scan. These outcomes from kalman filters (KF) are used to solve data association process and subsequently manage the tracks for each target.

\section{Update of Classifier probabilities}

A class-based re-sampling scheme is used to update the classifier probabilities in the form of joint feature measurement likelihoods.

\section{Algorithm for Update of Classifier Probabilities}

Input: C1, C2 and C3 cluster set for all trajectories. Output: Classifier Probability for each cluster

Step 1: initialize the window size as w[size]

Step 2: read all current cluster using below formula

$\mathrm{c}[$ set $]=\sum_{k=0}^{n}\left(\begin{array}{l}n \\ k\end{array}\right) x^{k-1}$

Step 3: if(Current_Node.valid(W[size]))

Step 4: Calculate probability for next target $\mathrm{T}=\mathrm{C}$ [set]

Step 5: define threshold as T

Validate target probability if $(\mathrm{T}[0]>=\mathrm{T})$

Step 6: Recommend T[0] as next target

Step 7: Read next set from $\mathrm{T}$ till null $\mathrm{T}[0] \leftarrow \mathrm{T}$ [current val]

Step 8: return $\mathrm{T}[0]$

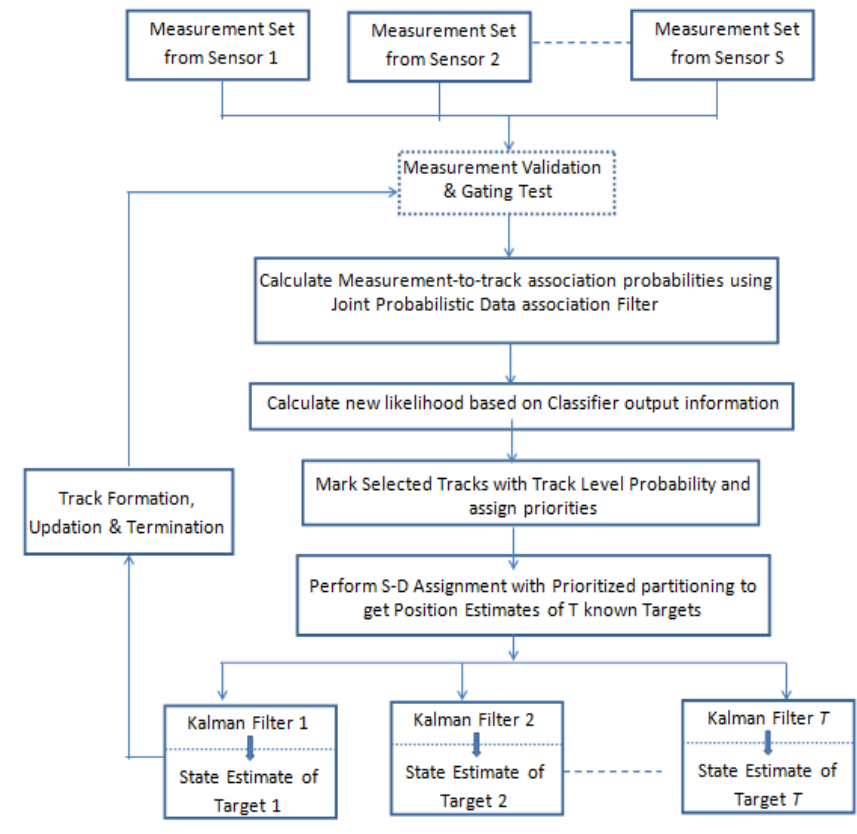

Fig. 4. Block Diagram of Data Association Using Proposed IMM Based Filter

\section{SIMULATION RESULTS}

\section{A. Simulation scenarios}

The algorithm is simulated under sparse non-maneuvering and maneuvering target scenarios in presence of clutter. No. of simulation performed with variable number of targets $=10$ (targets considered in simulations are moving with maneuvering and nonmaneuvering motion with clutter. Targets considered for simulation are closely spaced and crossed targets with conflicting scenarios.

For first 10 scans target move with high maneuvers with variable turn rate. Targets takes constant turns at known interval for next 10 scans. Few targets take maneuver motion and remaining targets take nonmaneuvering motion for next 5 scans (scan 20 to scan 25). Finally last 10 scans targets move with nonmaneuvering motion.

Simulation results up to 35 sensor data scans are analyzed in this section. Root Mean Square Errors (RMSE) and track loss using amount of deviation from true track with prior thresholds are used as to analyze the proposed methodology.

B. Results

Table- II: RMSEs for Position and Velocity Using Traditional IMM and Proposed IMM

\begin{tabular}{|c|c|c|c|c|}
\hline $\begin{array}{c}\text { Scan } \\
\text { No }\end{array}$ & \multicolumn{2}{|c|}{$\begin{array}{c}\text { RMSE Position Error } \\
\text { (km) }\end{array}$} & $\begin{array}{c}\text { RMSE Velocity Error } \\
\text { (km/s) }\end{array}$ \\
\cline { 2 - 5 } & $\begin{array}{c}\text { IMM } \\
\text { Algorithm }\end{array}$ & $\begin{array}{c}\text { IMM } \\
\text { Algorithm } \\
\text { with class } \\
\text { info. }\end{array}$ & $\begin{array}{c}\text { IMM } \\
\text { Algorithm }\end{array}$ & $\begin{array}{c}\text { IMM } \\
\text { Algorithm } \\
\text { with class } \\
\text { info. }\end{array}$ \\
\hline 5 & 0.186 & 0.043 & 0.034 & 0.157 \\
\hline 10 & 0.139 & 0.09 & 0.167 & 0.083 \\
\hline 15 & 0.097 & 0.041 & 0.097 & 0.039 \\
\hline 20 & 0.079 & 0.045 & 0.059 & 0.032 \\
\hline 25 & 0.067 & 0.053 & 0.062 & 0.036 \\
\hline 30 & 0.059 & 0.018 & 0.083 & 0.032 \\
\hline 35 & 0.062 & 0.023 & 0.085 & 0.036 \\
\hline
\end{tabular}


Table- III: Average Track Loss for Proposed IMM against traditional IMM

\begin{tabular}{|c|c|c|}
\hline $\begin{array}{c}\text { No. of } \\
\text { Targets }\end{array}$ & $\begin{array}{c}\text { Track Loss for } \\
\text { Traditional IMM } \\
\text { Algorithm }\end{array}$ & $\begin{array}{c}\text { Track Loss for } \\
\text { Proposed IMM } \\
\text { Algorithm with class } \\
\text { information }\end{array}$ \\
\hline 2 & $0 \%$ & $0 \%$ \\
\hline 4 & $0 \%$ & $0 \%$ \\
\hline 6 & $1 \%$ & $0 \%$ \\
\hline 8 & $3.2 \%$ & $2.3 \%$ \\
\hline 10 & $5 \%$ & $4.8 \% \%$ \\
\hline 15 & $6.4 \%$ & $3.75 \%$ \\
\hline 20 & $11 \%$ & $5.2 \%$ \\
\hline 25 & $22.3 \%$ & $10.56 \%$ \\
\hline
\end{tabular}

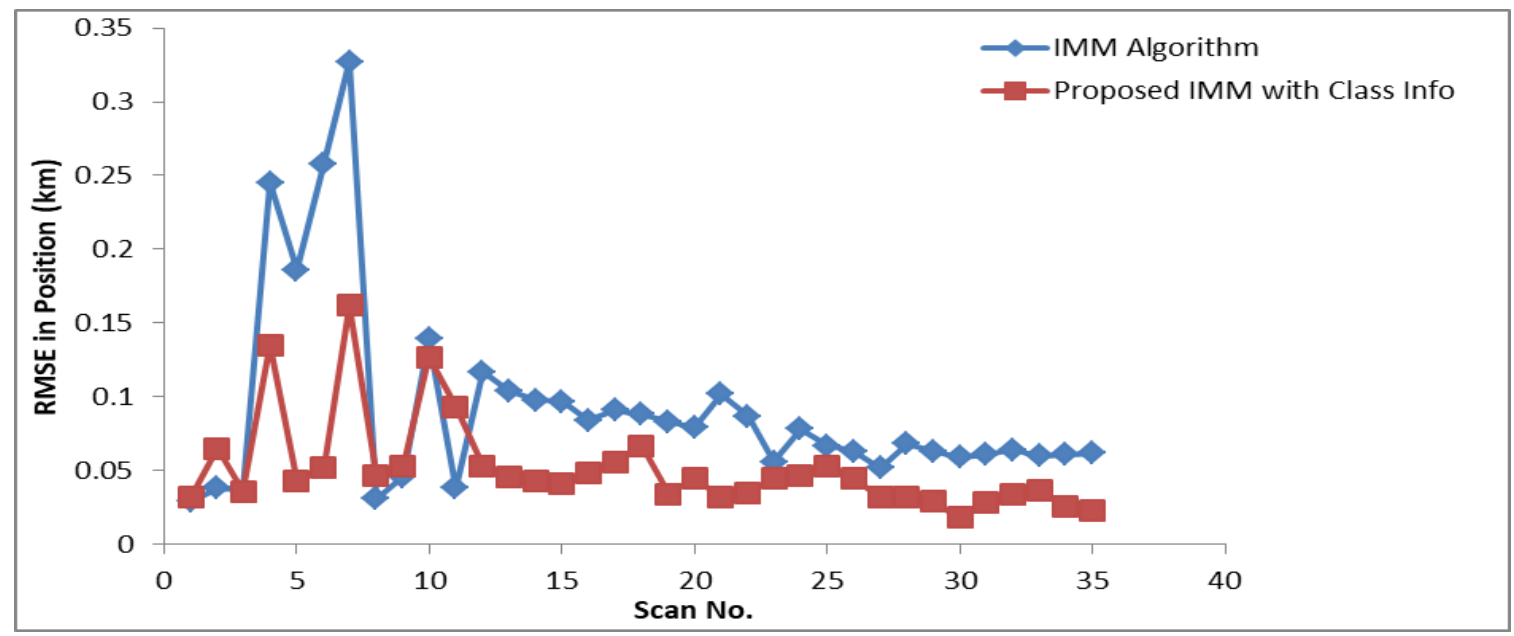

Fig. 5. RMSE of Estimated Positions for IMM and Proposed IMM with Class information

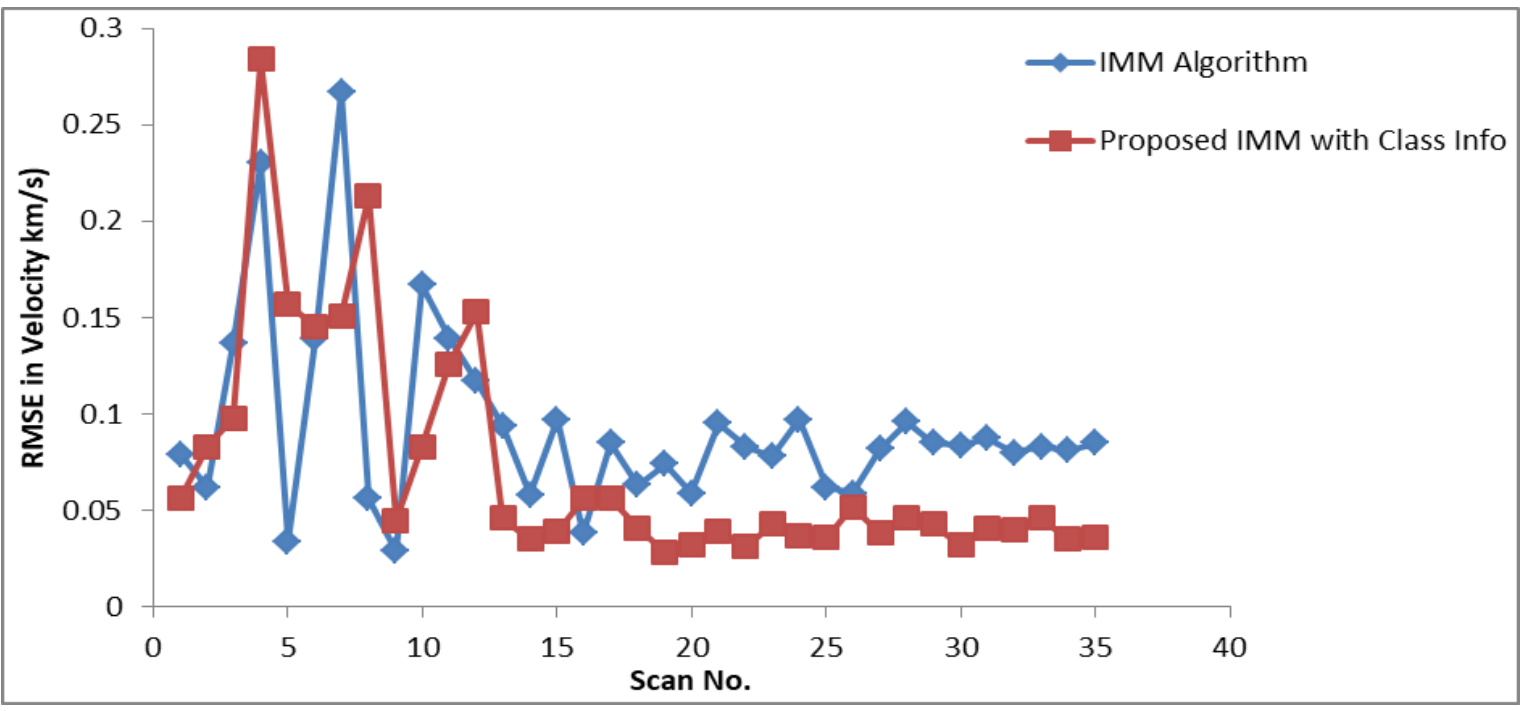

Fig. 6. RMSE of Estimated Velocities for IMM and Proposed IMM with Class information

Figure 5 and 6 demonstrates the position and speed RMSE in the diverse following situation. Plots comparing to non-moving and moving objective situations with various estimations of and are portrayed in these figures. It is clear that the RMSE esteems towards the finish of the situations for all following cases are decreasing prominently for proposed IMM algorithm.

\section{CONCLUSION}

The proposed algorithm for target tracking using classification information based data association filter use the target dynamic information more effectively in various tracking scenarios. It uses the classifier information in optimize way so that the similar target belongs to derived class can be track more capably as its motion model already decided in history scans. 
The synchronized use of target kinematic information and corresponding classification information during data association is used to derive more precise estimated tracks. In this paper these two strategies have been combined to get accurate tracking results. The proposed algorithm uses both target motion model and target classifier jointly to get optimized and accurate tracking results in presence of dens and cluttered environment. Algorithm also creates new tentative classes according to target behavior (motion) and update dynamic classifier to include these new classes and corresponding features in future scans.

\section{REFERENCES}

1. Wang X, Li T, Sun S, Corchado J. A survey of recent advances in particle filters and remaining challenges for multitarget tracking. Sensors. 2017;17(12):2707.

2. Lee EH, Zhang Q, Song TL. Markov Chain Realization of Joint Integrated Probabilistic Data Association. Sensors. 2017 Dec 10;17(12):2865.

3. Chen X, Li Y, Li Y, Yu J, Li X. A novel probabilistic data association for target tracking in a cluttered environment. Sensors. 2016 Dec 18;16(12):2180.

4. Kieritz H, Becker S, Hübner W, Arens M. Online multi-person tracking using integral channel features. InAdvanced Video and Signal Based Surveillance (AVSS), 2016 13th IEEE International Conference on 2016 Aug 23 (pp. 122-130). IEEE.

5. Tang S, Andres B, Andriluka M, Schiele B. Multi-person tracking by multicut and deep matching. InEuropean Conference on Computer Vision 2016 Oct 8 (pp. 100-111). Springer, Cham.

6. Tang S, Andriluka M, Andres B, Schiele B. Multiple people tracking by lifted multicut and person reidentification. InProceedings of the IEEE Conference on Computer Vision and Pattern Recognition 2017 Jul 1 (pp. 3539-3548).

7. Pan S, Bao Q, Chen Z. An Efficient TO-MHT Algorithm for Multi-Target Tracking in Cluttered Environment. . InProceedings of the IEEE Conference on Advanced Information Technology, Electronic and Automation Control, 2017

8. Coraluppi SP, Carthel CA. Multiple-Hypothesis Tracking for Targets Producing Multiple Measurements. IEEE Transactions on Aerospace and Electronic Systems. 2018 Jan 22.

9. Zhou R, Zhou K, Wu M, Teng J. Improved Interactive Multiple Models Based on Self-Adaptive Turn Model for Maneuvering Target Tracking. In2018 Eighth International Conference on Information Science and Technology (ICIST) 2018 Jun 30 (pp. 450-457). IEEE.

10. Liu M, Fan Z, Wang X, Zhang S. IMM algorithm based on $H \infty$ filter for maneuvering target tracking. InControl Conference (CCC), 2017 36th Chinese 2017 Jul 26 (pp. 5385-5391). IEEE.

11. Cai L, Xu X, Liu J, Mo L, Tang J. An IMM Algorithm for Tracking Maneuvering Targets Based on Current Statistical Model. InComputational Intelligence and Design (ISCID), 2016 9th International Symposium on 2016 Dec 10 (Vol. 1, pp. 8-11). IEEE.

12. Vasuhi S, Vaidehi V. Target tracking using interactive multiple model for wireless sensor network. Information Fusion. 2016 Jan 1;27:41-53.

13. Hao N, Chu K, Chang T, Zhang L. A Moving State Estimation Method Based on Interactive Multi-Model Algorithm. In2018 2nd IEEE Advanced Information Management, Communicates, Electronic and Automation Control Conference (IMCEC) 2018 May 25 (pp. 2164-2167). IEEE.

14. Sun M, Ma Z, Li Y. Maneuvering target tracking using IMM kalman filter aided by elman neural network. InIntelligent Human-Machine Systems and Cybernetics (IHMSC), 2015 7th International Conference on 2015 Aug 26 (Vol. 1, pp. 144-148). IEEE.

15. Li B, Pang F, Liang C, Chen X, Liu Y. Improved interactive multiple model filter for maneuvering target tracking. InControl Conference (CCC), 2014 33rd Chinese 2014 Jul 28 (pp. 7312-7316). IEEE.

16. Amrouche N, Khenchaf A, Berkani D. Multiple target tracking using track before detect algorithm. InElectromagnetics in Advanced Applications (ICEAA), 2017 International Conference on 2017 Sep 11 (pp. 692-695). IEEE.

17. Coraluppi S, Carthel C. Track Management in Multiple-Hypothesis Tracking. In2018 IEEE 10th Sensor Array and Multichannel Signal Processing Workshop (SAM) 2018 Jul 8 (pp. 11-15). IEEE.
18. He S, Shin HS, Tsourdos A. Track-Oriented Multiple Hypothesis Tracking Based on Tabu Search and Gibbs Sampling. IEEE Sensors Journal. 2017 Oct 2;18(1):328-39.

19. Zhou Y, Li J. Interactive multiple Kalman filters based 3-D target tracking for two airborne platforms with bearings-only measurements. InControl and Decision Conference (CCDC), 2012 24th Chinese 2012 May 23 (pp. 2549-2553). IEEE.

20. Zhen C, Liu S, Wang J, Li J. A multiple attribute decision algorithm for single-object benchmark. InControl and Decision Conference (CCDC), 2016 Chinese 2016 May 28 (pp. 3912-3917). IEEE.

21. Ding R, Yu M, Oh H, Chen WH. New multiple-target tracking strategy using domain knowledge and optimization. IEEE Transactions on Systems, Man, and Cybernetics: Systems. 2017: 47(4): 605-616.

22. Dondo DG, Redolfi J, Griffa M, Steiner G, Canali L. Target tracking system using multiple cameras and Bayesian estimation. In2015 XVI Workshop on Information Processing and Control (RPIC) 2015 Oct 6 (pp. 1-6). IEEE.

23. Coraluppi SP, Carthel CA. Multiple-Hypothesis Tracking for Targets Producing Multiple Measurements. IEEE Transactions on Aerospace and Electronic Systems. 2018 Jan 22.

24. Cao X, Jiang X, Li X, Yan P. Correlation-based tracking of multiple targets with hierarchical layered structure. IEEE transactions on cybernetics. 2018 Jan;48(1):90-102.

25. 25.Mei W, Xiong G, Gong J, Yong Z, Chen H, Di H. Multiple moving target tracking with hypothesis trajectory model for autonomous vehicles. In Intelligent Transportation Systems (ITSC), 2017 IEEE 20th International Conference on 2017 Oct 16 (pp. 1-6). IEEE.

26. Li D, Zhang Y, Lin Y, Liu J. A novel track initiation method for track splitting and merging. In OCEANS 2016-Shanghai 2016 Apr 10 (pp. 1-7). IEEE.

27. Murthy KG. An algorithm for ranking all the assignments in order of increasing costs. Operations research. 1968;16(3):682-7.

28. Lu B, Rosenbaum PR. An Algorithm for Ranking all the Assignments in Order of Increasing Cost. Journal of Computational and Graphical Statistics. 2004;13(2):422-34.

29. Pedersen CR, Nielsen LR, Andersen KA. An algorithm for ranking assignments using reoptimization. Computers \& Operations Research. 2008 Nov 1;35(11):3714-26.

30. Wang F. Fuzzy supervised classification of remote sensing images. IEEE Transactions on geoscience and remote sensing. 1990 Mar;28(2):194-201.

31. Yang W, Fu Y, Li X. Joint target tracking and classification via RFS-based multiple model filtering. Information Fusion. 2014 Jul 1;18:101-6.

32. Thite, A. \& Mishra, A., 2016, February. Optimized multi-sensor multi-target tracking algorithm for air surveillance system. In 2016 2nd International Conf on Advances in Electrical, Electronics, Information, Communication and Bio-Informatics (AEEICB) (pp. 637-642). IEEE.

\section{AUTHORS PROFILE}

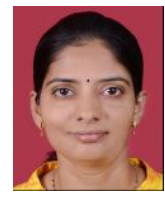

Anita Thite, received the master degree in Computer Engineering from Pune Institute of Advanced technology, Pune, India. She is currently the Ph.D. research scholar of the department of Computer Science and Engineering, Defence Institute of Advanced Technology, Pune, India Her research interests include Advance Algorithms, Problem Solving Techniques, Target Tracking and Data Association, High Performance Computing, etc.

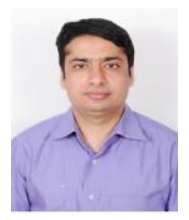

Arun Mishra, received M.Tech and $\mathrm{PhD}$ degree in Computer engineering from MNIT, Allahabad, India. He is currently working as an Assistant Professor in the department of Computer Science and Engineering, Defence Institute of Advanced Technology, Pune, India His research interests include Secure Software Engineering, Trusted Computing, Operating System, Object Oriented Analysis \& Design, Algorithms, Data Association. 